\title{
Comparison of Platelet Count Reduction in Patients awaiting Liver Transplantation with and without Primary Sclerosing Cholangitis: A Cohort Study
}

\author{
Mohssen Nassiri Toosi, MD $^{1 *}$; Bobak Moazzami, MD ${ }^{1 *}$; Ali Jafarian, MD $^{1}$; Amirhossein Emami, MD'; Farid Azmodeh Ardalan, MD'; Mehrdad \\ Karimi, $\mathrm{PhD}^{1}$
}

'Liver Transplantation Research Center, Tehran University of Medical Sciences, Tehran, Iran

\begin{abstract}
Background: Thrombocytopenia is the most well-known hematological abnormality occurring in patients with liver cirrhosis. However, the rate of platelet count reduction is not the same across different chronic liver disease etiologies. Therefore, the aim of the present study was to compare the differences in the platelet count levels between primary sclerosing cholangitis-related cirrhosis (PSC-C) and other causes of liver disease.

Methods: In this cohort study, the association between PSC-C and risk of platelet count reduction was investigated. The platelet counts were repeatedly measured among 242 consecutive cirrhotic patients (144 males and 98 females) including 67 patients with PSC-C and 175 patients with non-PSC-C who were on the waiting list for liver transplantation. The Poisson regression analysis was used to assess the relationship between platelet count reduction and PSC-C, after adjusting for potential confounding factors. Results: During the five years of follow-up, comparison between the two groups revealed that significantly higher levels of platelet were found in PSC-C patients when compared to the non-PSC-C group [148 $(106-280)\left(\times 10^{3} / \mu \mathrm{L}\right)$ vs. $79(50-110)\left(\times 10^{3} / \mu \mathrm{L}\right)$, respectively, $P<0.001]$. After adjusting for confounding factors, a significant association was observed between non-PSC-C and the risk of platelet count reduction (relative risk, RR: $14.81,95 \% \mathrm{Cl}: 1.21-160.42 ; P=0.03$ ).

Conclusion: The findings indicate that PSC-C patients present with mild degrees of thrombocytopenia compared to other causes of chronic liver disease.

Keywords: Liver cirrhosis, Liver transplantation, Primary sclerosing cholangitis, Thrombocytopenia

Cite this article as: Nassiri Toosi M, Moazzami B, Jafarian A, Emami A, Azmodeh Ardalan F, Karimi M. Comparison of platelet count reduction in patients awaiting liver transplantation with and without primary sclerosing cholangitis: a cohort study. Arch Iran Med. 2020;23(11):732-739. doi: 10.34172/aim.2020.97.
\end{abstract}

Received: June 30, 2019, Accepted: September 6, 2020, ePublished: November 1, 2020

\section{Introduction}

Liver cirrhosis (LC) is the result of diffuse processes of liver cell necrosis, replacement by extensive fibrosis, liver cell regeneration and subsequent development of regenerative nodules. ${ }^{1}$ Patients with LC, regardless of their etiology, develop changes in their hemostatic parameters including decreased levels of platelet counts. ${ }^{2-5}$ Thrombocytopenia is defined as a platelet count less than $150 \times 10^{3} / \mu \mathrm{L}$, and occurs in $6 \%-84 \%$ of patients with LC. ${ }^{6-8}$ Various theories have been proposed regarding the causes of thrombocytopenia in cirrhotic patients such as reduced levels of thrombopoietin, sequestration of platelets in the spleen due to portal hypertension, destruction of platelets as a result of autoantibodies and secondary bone marrow suppression due to underlying liver disease. ${ }^{9-17}$ Although thrombocytopenia is believed to be an indicator of advanced liver disease, it has also been used as a marker of prognosis..$^{18}$ However, the rate of platelet count reduction appears to be different among various etiologies of liver disease. ${ }^{19}$

Primary sclerosing cholangitis (PSC) refers to a heterogeneous, rare, cholestatic liver disorder with unknown underlying pathogenesis. ${ }^{20,21}$ Despite increased knowledge regarding certain aspects of PSC, the exact pathophysiology of its progression to cirrhosis remains largely unknown. Currently, there are no established laboratory tests for differentiating PSC from other causes of LC and its diagnosis often requires a multidisciplinary approach. ${ }^{22}$ Several studies have indicated that patients with PSC often show distinct laboratory features such as a hypercoagulable state seen in cholestatic liver disease rather than non-cholestatic etiologies. ${ }^{23,24}$ However, these studies have not assessed platelet count differences between PSC and non-PSC etiologies.

Given the diverse nature of platelet count changes in different liver disease etiologies, and since there was no large study regarding platelet count changes in patients with a diagnosis of PSC-related cirrhosis (PSC-C), we

*Corresponding Authors: Mohssen Nassiri Toosi, MD; Department of Liver Transplantation, Imam Khomeini Hospital, Keshavarz Blvd, Tehran, Iran. Tel: +982166581598; Fax: +982166581521; Email: nasirito@sina.tums.ac.ir;

Bobak Moazzami, MD; Liver Transplantation Research Center, Tehran University of Medical Sciences, Tehran, Iran. Tel: +982122413482; Fax: +982122174723; Email: Moazzami.bobak@gmail.com 
conducted this observation among LC patients (PSC-C and non-PSC-C) who were on the liver transplantation waiting list.

\section{Materials and Methods \\ Patient Population}

The present cohort study was performed on all consecutive cirrhotic patients who were on the waiting list for liver transplantation in a liver transplantation program (affiliated to Tehran University of Medical Sciences, a tertiary referral center for chronic liver disease) from March 2013 to January 2017. Data regarding patients' demographic, clinical and hematological characteristics were obtained. The diagnosis of PSC was made after the exclusion of a variety of other causes of cholestatic liver disease and secondary sclerosing cholangitis, as well as the combination of prolonged cholestasis and imaging modalities of magnetic resonance cholangiopancreatography or endoscopic retrograde cholangiopancreatography indicating the presence of bile duct changes with multifocal strictures and "beaded" appearance..$^{20,25,26}$ According to the European Association for the Study of the Liver guidelines, the diagnosis of other cirrhosis etiologies was established. ${ }^{27}$ Patients with a prior history of splenectomy, incomplete hematologic assessment, cholangiocarcinoma, recent cholangitis, use of any medications that might influence the platelet counts (Interferon, Azathioprine, Cellcept), or those who received platelet transfusion before enrollment were excluded from the study. The severity of LC was assessed using Child-Pugh and Model for End-Stage Liver Disease (MELD) scores. The study protocol was approved by the ethics committee of Tehran University of Medical Science. Informed consent was obtained from all included patients. The study was conducted in accordance with the Declaration of Helsinki and other applicable guidelines, laws, and regulations. ${ }^{28}$

\section{Measurements}

Repeated measurements of standard routine laboratory tests including white-blood cell count (WBC), and hemoglobin, platelets, creatinine, international normalized ratio (INR), aspartate aminotransferase (AST), alanine aminotransferase (ALT), alkaline phosphatase (ALP), gamma-glutamyltransferase (GGT), and erythrocyte sedimentation rate (ESR) were collected. Platelet count was measured in EDTA-treated blood samples collected in 2-3 h, using Sysmex's unique fluorescence flow cytometry (the results was confirmed by double-checking or using the peripheral blood smear for each analysis).

Patients were divided into four groups based on the MELD score quartiles: 1) MELD score <12; 2) MELD score 12-15; 3) MELD score 15-20; and 4) MELD score $>20$. Spleen size was evaluated based on ultrasonography, and its largest diameter was classified into three groups (group 1: <120 mm, group 2: 120-150 mm, group 3:
$>150 \mathrm{~mm}$ ). Portal vein size was determined either by CT scan or ultrasonography, and categorized into three groups according to the mean diameter of the portal size $(<12$, $12-14$ and $>14 \mathrm{~mm}$ ). Esophageal variceal (EV) grading was assessed based on the Japanese classification including: F0 (no EV detected), F1 (small or less than $5 \mathrm{~mm}$ straight EV), F2 (slightly enlarged tortuous EV occupying less than one-third of the esophageal lumen) and F3 (large coil-shaped EV that occupied more than one-third of the esophageal lumen). ${ }^{29}$

\section{Statistical Analysis}

Nominal and ordinal variables were reported as count $(\%)$, and continuous numeric variables were reported as mean \pm standard deviation (SD) or the median and 25 th and 75 th percentiles if non-normally distributed. We estimated whether data were distributed normally using Kolmogorov-Smirnov and Shapiro-Wilk tests, as well as Q-Q plot. Parametric and non-parametric analyses were used according to the finding of these tests. Pearson correlation was used for normally distributed data and Spearman rank correlation was used for non-normally distributed data. Data on the diagnosis of PSC vs nonPSC, as well as platelet counts and other hematologic parameters including age, sex, and MELD score, were available for all patients. Five percent of the population did not have data on EV bleeding, 8\% did not have data on diuretic intake, and $9 \%$ did not have data on hepatic encephalopathy.

The incidence of different levels of decreased platelet count was calculated for the PSC-C and non-PSC-C groups. With the sample size of the present study, at a significance level of 5\%, we were able to detect a difference in platelet counts of at least $10 \%$ with $99 \%$ power. Poisson regression analysis was performed in order to test the association between platelet count reduction and diagnosis of PSC-C by calculating the relative risk (RR) and 95\% confidence interval (CI). The goodness-of-fit of the model was assessed by Hosmer-Lemeshow test. A directed acyclic graph was used to assist in the selection of appropriate covariates (confounder and competing exposure) by modeling the relationships between potential covariates, PSC state and platelet count reduction (Figure S1, see Supplementary file 1). Confounding factors including gender, age, WBC, ESR, cholesterol, MELD score, portal size and spleen size were included as covariates in the multivariable models. These variables were included given that they were individually significantly associated with both platelet count and diagnosis of PSC. Statistical significance was defined as $P<0.05$. Data were analyzed using SPSS 23.0 (SPSS Inc.; Chicago, IL).

\section{Results}

Characteristics of Patients

A total of 242 cirrhotic patients were included in this 
study. Table 1 describes the baseline demographic and biochemical data. The mean age of patients was $37.2 \pm$ 14.1 and $42.1 \pm 14.2$ years for patients with PSC-C and non-PSC-C, respectively. The most common etiology of LC in this study was PSC which was found in 67 patients (27.6\%) followed by cryptogenic (23.1\%), Hepatitis B (16.5\%), autoimmune hepatitis (12.8\%), Hepatitis C (8.7\%), Wilson (4.1\%) and primary biliary cirrhosis (2.5\%). Compared to non-PSC-C patients, the PSC-C patients were younger $(P=0.016)$. In both groups, no significant difference was found in mean age between males and females $(P=0.2)$. The MELD score was similar in both groups (PSC-C, $15.6 \pm 4.2$ vs. non-PSC-C, 16.7 $\pm 5.7)$.
Association between PSC and Platelet Count Reduction The comparison between the two groups revealed a significant difference in platelet count reduction $(P<0.001)$. Platelet counts were significantly reduced from a median of $148(106-280)\left(\times 10^{3} / \mu \mathrm{L}\right)$ at baseline to $140(97-260)\left(\times 10^{3} / \mu \mathrm{L}\right)$ at the follow-up in the PSC-C group $(P<0.001)$. Similarly, a reduction in platelet count in the non-PSC-C group was observed from a median of $79(50-110)$ at baseline to $65(45-90)$ at the follow-up period $(P<0.001)$. A platelet count reduction more than $20 \%$ from baseline $\left(150 \times 10^{3} / \mu \mathrm{L}\right)$ was seen in $37 / 67$ and $171 / 175$ patients with PSC-C and non-PSC-C diagnosis, respectively. The reduction in platelet count was significantly higher in the non-PSC-C compared to

Table 1. Baseline Characteristics of 242 Cirrhotic Patients

\begin{tabular}{|c|c|c|c|c|}
\hline Demographics & PSC-C $(n=67)$ & Non-PSC-C $(n=175)$ & Total & $P$ \\
\hline Age (year) $( \pm S D)$ & $37.40 \pm 13.81$ & $42.12 \pm 14.22$ & $40.81 \pm 14.24$ & $0.02^{*}$ \\
\hline Gender & & & & 0.41 \\
\hline Male (\%) & $37(55.2)$ & $107(61.1)$ & $144(59.9)$ & \\
\hline Female (\%) & $30(44.8)$ & $68(38.9)$ & $98(40.5)$ & \\
\hline Platelet $\left(\times 10^{3} / \mu \mathrm{L}\right)(\mathrm{IQR})$ & $148(174)$ & $79(60)$ & $91(74)$ & $0.001^{* *}$ \\
\hline WBC $\left(/ \mathrm{mm}^{3}\right)(\mathrm{IQR})$ & $4200(1800)$ & $3000(1300)$ & $3500(2300)$ & $0.001^{* *}$ \\
\hline $\mathrm{Hb}(\mathrm{g} / \mathrm{dL})$ & $10.56 \pm 1.85$ & $11.29 \pm 1.85$ & $11.10 \pm 1.88$ & $0.007^{*}$ \\
\hline $\operatorname{ESR}(\mathrm{mm} / \mathrm{h})(\mathrm{IQR})$ & $30.5(58)$ & $20(25)$ & $21(34.8)$ & $0.016^{* *}$ \\
\hline $\mathrm{MCV}$ & $87.53 \pm 12.26$ & $88.21 \pm 9.05$ & $88.03 \pm 10.03$ & $0.683^{*}$ \\
\hline MCV > $95(\%)$ & 38.5 & 32 & & \\
\hline INR (IQR) & $1.4(0.4)$ & $1.7(0.3)$ & $1.6(0.5)$ & $0.001^{* *}$ \\
\hline Ferritin (IQR) & $50.5(83.4)$ & $61.5(136.4)$ & $54(124)$ & $0.427^{* *}$ \\
\hline Cholesterol (mg/dL) & $183.01 \pm 74.84$ & $141.47 \pm 34.79$ & $152.75 \pm 52.18$ & $0.001^{*}$ \\
\hline $\mathrm{LDL}(\mathrm{mg} / \mathrm{dL})$ & $114.26 \pm 58.76$ & $78.10 \pm 27.56$ & $89.35 \pm 33.16$ & $0.001^{*}$ \\
\hline $\mathrm{TG}(\mathrm{mg} / \mathrm{dL})(\mathrm{IQR})$ & $90(74.2)$ & $70(39.3)$ & $81.4(42.7)$ & $0.008^{* *}$ \\
\hline $\mathrm{HDL}(\mathrm{mg} / \mathrm{dL})$ & $41.35 \pm 20.21$ & $43.78 \pm 14.90$ & $43.04 \pm 16.70$ & $0.442^{*}$ \\
\hline Albumin (g/dL) & $3.37 \pm 0.57$ & $3.43 \pm 0.59$ & $3.42 \pm 0.59$ & $0.473^{*}$ \\
\hline Creatinine (mg/dL) (IQR) & $0.8(0.2)$ & $1(0.3)$ & $0.9(0.3)$ & $0.001^{* *}$ \\
\hline Total bilirubin (mg/dL) (IQR) & $5.8(10.2)$ & $2.5(2)$ & $2.8(3.5)$ & $0.001^{* *}$ \\
\hline Portal size diameter (mm) & $12.68 \pm 3.07$ & $13.58 \pm 2.95$ & $13.33 \pm 3.00$ & $0.081^{*}$ \\
\hline Spleen size $(\mathrm{mm})$ & $165.45 \pm 41.28$ & $156.61 \pm 37.47$ & $158.94 \pm 38.61$ & $0.146^{*}$ \\
\hline \multicolumn{5}{|l|}{ Esophageal varices (\%) } \\
\hline F0 & $13(26.5)$ & $14(9.2)$ & $27(13.4)$ & \multirow{4}{*}{$0.007^{*}$} \\
\hline $\mathrm{F} 1$ & $11(22.4)$ & $48(31.4)$ & $59(29.2)$ & \\
\hline $\mathrm{F} 2$ & $16(32.7)$ & $42(27.5)$ & $58(28.7)$ & \\
\hline F3 & $9(18.4)$ & $49(32)$ & $58(28.7)$ & \\
\hline MELD & $16.7 \pm 5.7$ & $15.6 \pm 4.2$ & $15.58 \pm 5.10$ & $0.021^{* *}$ \\
\hline Child-Pugh (IQR) & $9(3)$ & $8(2)$ & $8(3)$ & $0.25^{* *}$ \\
\hline Esophageal variceal bleeding (\%) & $16(23.8)$ & $55(31.4)$ & $71(29.3)$ & 0.348 \\
\hline Diuretic intake H. (\%) & $18(23.8)$ & $55(31.4)$ & $73(30.1)$ & 0.910 \\
\hline SBP Hx. (\%) & 1.5 & 5.1 & & 0.2 \\
\hline Hepatic encephalopathy Hx. (\%) & $38(56.7)$ & $100(57.1)$ & $138(57)$ & 0.103 \\
\hline IBD Hx. (\%) & $43(64.1)$ & $1(0.5)$ & $44(18.1)$ & 0.001 \\
\hline Colectomy Hx. (\%) & $15(22.3)$ & $1(0.5)$ & $16(6.6)$ & 0.1 \\
\hline PVT Hx. (\%) & $3(4.4)$ & $12(6.8)$ & $15(6.1)$ & 0.610 \\
\hline Comorbid disease (\%) & $5(33.3)$ & $25(28.1)$ & $30(28.8)$ & 0.678 \\
\hline
\end{tabular}

Data are presented as Mean \pm SD. IQR: Inter quartile range with $75^{\text {th }}$ and $25^{\text {th }}$ percentiles.

PSC-C, Primary sclerosing cholangitis related cirrhosis; WBC, white blood cell; Hb, hemoglobin; ESR, Erythrocyte sedimentation rate; MCV, Mean corpuscular volume; LDL, Low-density lipoprotein; TG, Triglycerides; HDL, High-density lipoprotein; MELD, Model for end stage liver disease; UTI, Urinary tract infection; PVT, portal vein thrombosis; SBP, Spontaneous bacterial peritonitis.

$P<0.05$ were considered significant.

Between-group comparison was made using independent $t$ test.

“* Between-group comparison was made using Mann-Whitney's $U$ test. 
PSC-C group $(P<0.001)$.

Unadjusted Poisson regression analysis indicated that patients in the non-PSC-C group compared to those with PSC-C had a relative risk (RR) of 28.14 (95\% CI 7.45-88.10) for having an increased risk of platelet count reduction during the follow-up period $(P<0.001)$. After adjusting for age, WBC, ESR, cholesterol, portal size, and spleen size, this association was still statistically significant in non-PSC-C (RR: 14.81, 95\% CI: 1.21-160.42; $P=$ 0.03) (Table 2). The Hosmer-Lemeshow $\chi 2$ statistic showed that the goodness-of-fit was good for this model $(\chi 2=5.22, P=0.73)$

Association between Platelet Count and LC Etiologies As shown in Figure 1, a significant difference was found in platelet count levels between different etiologies of cirrhosis $(P<0.001)$ (Table 3$)$. Post hoc test results indicated that the platelet count was higher in PSC-C patients compared to other etiologies of $\mathrm{LC}(P<0.001)$.

Association between Platelet Count and ESR Levels The median ESR levels were significantly higher in PSC-C patients compared to the non-PSC-C group (30.5 vs. 20,

Table 2. Multivariable Poisson Regression Analysis on the Association Between Non-PSC-C and Risk of Platelet Count Reduction

\begin{tabular}{llll}
\hline Covariates & $\mathbf{R R}$ & $\mathbf{9 5} \% \mathbf{C I}$ & $\boldsymbol{P}$ \\
\hline Non-PSC-C diagnosis & 14.81 & $1.21-160.42$ & 0.03 \\
Age & 1.009 & $0.88-1.07$ & 0.38 \\
WBC $\left(/ \mathrm{mm}^{3}\right)$ & 0.98 & $0.97-1.00$ & 0.02 \\
\hline Cholesterol $(\mathrm{mg} / \mathrm{dL})$ & 1.002 & $0.98-1.01$ & 0.31 \\
\hline Portal size $(\mathrm{mm})$ & 1.11 & $0.66-1.75$ & 0.31 \\
\hline Spleen size $(\mathrm{mm})$ & 1.009 & $0.98-1.03$ & 0.35 \\
\hline
\end{tabular}

PSC-C, Primary sclerosing cholangitis related cirrhosis; WBC, White blood cell; $\mathrm{RR}$, relative risk; $\mathrm{Cl}$, confidence interval.

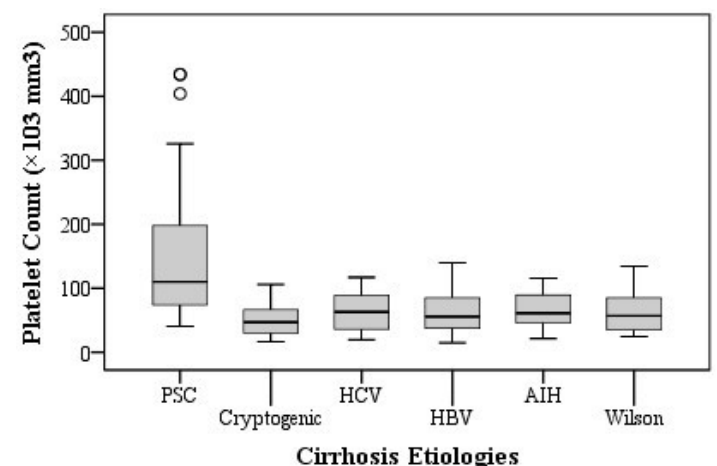

Figure 1. Association between Platelet Count and Liver Cirrhosis Etiologies. Distribution of platelet indices in the 5 groups: PSC, cryptogenic, hepatitis C virus (HCV), hepatitis B virus (HBV), autoimmune hepatitis (AlH) and Wilson. The boxes represent the interquartile range (IQR), with the upper and lower edges of the boxes representing the 75th and 25th percentiles, respectively. The central horizontal lines within the boxes represent the median levels for each group.
$P=0.016$ ) (Table 1). Moreover, there was a significant positive correlation between platelet counts and ESR levels $(r=0.318, P<0.001)$.

Comparison of Platelet Count in Different MELD Categories

After stratification of MELD score into 4 quartiles $(<12$, $12-15,15-20$ and $>20$ ), a comparison of platelet count between PSC-C and non-PSC-C patients revealed that platelet count was significantly different $(P<0.001)$ (Figure 2A). Also, in different MELD quartiles, the platelet count remained higher in the PSC-C group compared to the non-PSC-C group $(P<0.001)$ Moreover, the MELD category did not differ in PSC-C patients $(P=0.80)$, however, in the non-PSC-C group, MELD category was different based on platelet count $(P=0.004)$.

Comparison of Platelet Count in Different Variceal Grades We found that there was a significant association between the grade of esophageal varices and platelet count in PSC-C patients $(P=0.048)$ (Figure $2 \mathrm{~B})$. In contrast, when the group with non-PSC-C was assessed, no association was observed between serum platelet count and variceal grade $(P=0.68)$ (Table 3$)$. Moreover, among all variceal grades, platelet counts were higher in the PSC-C group compared to non-PSC-C patients $(P<0.001)$.

\section{Comparison of Platelet Count in Different Spleen Size} Categories

The results of comparing platelet counts between PSC-C and non-PSC-C across different spleen size categories indicated that in all spleen groups, there were higher platelet counts in PSC-C than non-PSC-C $(P<0.001)$ (Figure 2C). In both PSC-C and non-PSC-C groups, a significant negative correlation existed between platelet counts and spleen size $(r=-0.43, P=0.001$ vs $r=-0.34$, $P<0.001)$.

Comparison of Platelet Count in Different Portal Vein Size Categories

The results showed that platelet counts were significantly different between PSC-C and non-PSC-C groups across all portal size categories $(P<0.01)$ (Figure $2 \mathrm{D})$. There was also a negative correlation between platelet count and portal vein size in the non-PSC-C group $(r=0.19$; $P=0.038)$. On the other hand, in the PSC-C group, no significant correlation was observed between portal size and platelet count $(\mathrm{r}=0.18, P=0.22)$.

\section{Discussion}

In the present study, we evaluated patients' platelets counts in relation to the spectrum of chronic liver disease etiologies. Our findings revealed that the platelet count reduction was significantly higher in cirrhotic patients with non-PSC-C when compared to patients with a 
Table 3. A Comparison of Platelet Count $\left(\times 10^{3} / \mu \mathrm{L}\right)$ Levels between PSC-C and Non-PSC-C Patients

\begin{tabular}{|c|c|c|c|c|}
\hline & \multicolumn{3}{|c|}{ Cirrhosis Groups } & \multirow{2}{*}{$P$ Value } \\
\hline & Total & PSC-C & Non-PSC-C & \\
\hline MELD & & & & 0.002 \\
\hline$<12$ & $93.0(62.5-111.0)$ & $143.0(90.7-275.0)$ & $83.0(58.5-101.5)$ & \\
\hline $12-15$ & $61.0(41.5-81.0)$ & $145.0(57.0-255.5)$ & $56.0(38.2-79.0)$ & \\
\hline $15-20$ & $64.0(46.0-96.0)$ & $104.0(83.0-142.5)$ & $57.0(38.0-83.0)$ & \\
\hline$>20$ & $80.5(44.0-120.2)$ & $117.5(67.5-184.7)$ & $44.5(30.0-83.5)$ & \\
\hline Portal size (mm) & & & & $<0.001$ \\
\hline$<12$ & $74.0(55.2-113.5)$ & $119(119-219)$ & $62.5(46.0-88.5)$ & \\
\hline $12-14$ & $64.5(42.2-98.0)$ & 105 (96-180) & $51.0(39.5-84.5)$ & \\
\hline$>14$ & $61.0(39.5-82.5)$ & $76(63.5-116.7)$ & $51(30-70)$ & \\
\hline Variceal grade & & & & $<0.001$ \\
\hline F0 & $79(57-192)$ & $192(92-270)$ & $59.5(38.2-70.0)$ & \\
\hline $\mathrm{F} 1$ & 70 (45-98) & $106(78-126)$ & $57.0(36.7-33.7)$ & \\
\hline F2 & $64(44-93.5)$ & $81(60-164)$ & $56.5(39.5-87.2)$ & \\
\hline F3 & $55.5(30.0-86.2)$ & $96(63-107)$ & $51.0(30.0-81.0)$ & \\
\hline Spleen size $(\mathrm{mm})$ & & & & $<0.001$ \\
\hline$<120$ & $106.5(74.2-240.0)$ & $260.0(132.7-283.2)$ & $82.5(60.2-106.7)$ & \\
\hline $120-150$ & $68.0(48.2-99.5)$ & $110.0(65.0-145.0)$ & $61.0(46.5-91.0)$ & \\
\hline$>150$ & $61.0(39.5-84.0)$ & $85.0(66.5-145.0)$ & $46.0(30.0-70.0)$ & \\
\hline
\end{tabular}

MELD, Model for end-stage liver disease; PSC-C, Primary sclerosing cholangitis-related cirrhosis.

diagnosis of PSC.

Coagulation disorders are closely linked to progressive liver failure and account for adverse outcomes among cirrhotic patients. ${ }^{30}$ Several studies have shown that the degree of thrombocytopenia is different in various chronic liver disease. Tejima et $\mathrm{al}^{31}$ reported that patients with hepatitis C-related hepatocellular carcinoma and advanced LC had worsening degrees of thrombocytopenia compared to those with hepatitis B-related hepatocellular carcinoma. They suggested that the underlying mechanism for the greater levels of thrombocytopenia in patients with hepatitis C-related cirrhosis may be due to impaired platelet production rather than platelet destruction. Another study demonstrated that patients with hepatitis C-related cirrhosis had significantly lower platelet counts compared to cirrhotic patients with non-alcoholic fatty liver disease etiology. ${ }^{32}$ In the current study, we found significantly higher levels of platelet count in PSC-C patients compared to other cirrhotic etiologies. To the best of our knowledge, this is the first study to delineate the platelet count differences between patients with PSC-C and non-PSC-C diagnosis.

Previous studies have shown that bleeding complications in patients with advanced PSC are much less frequent
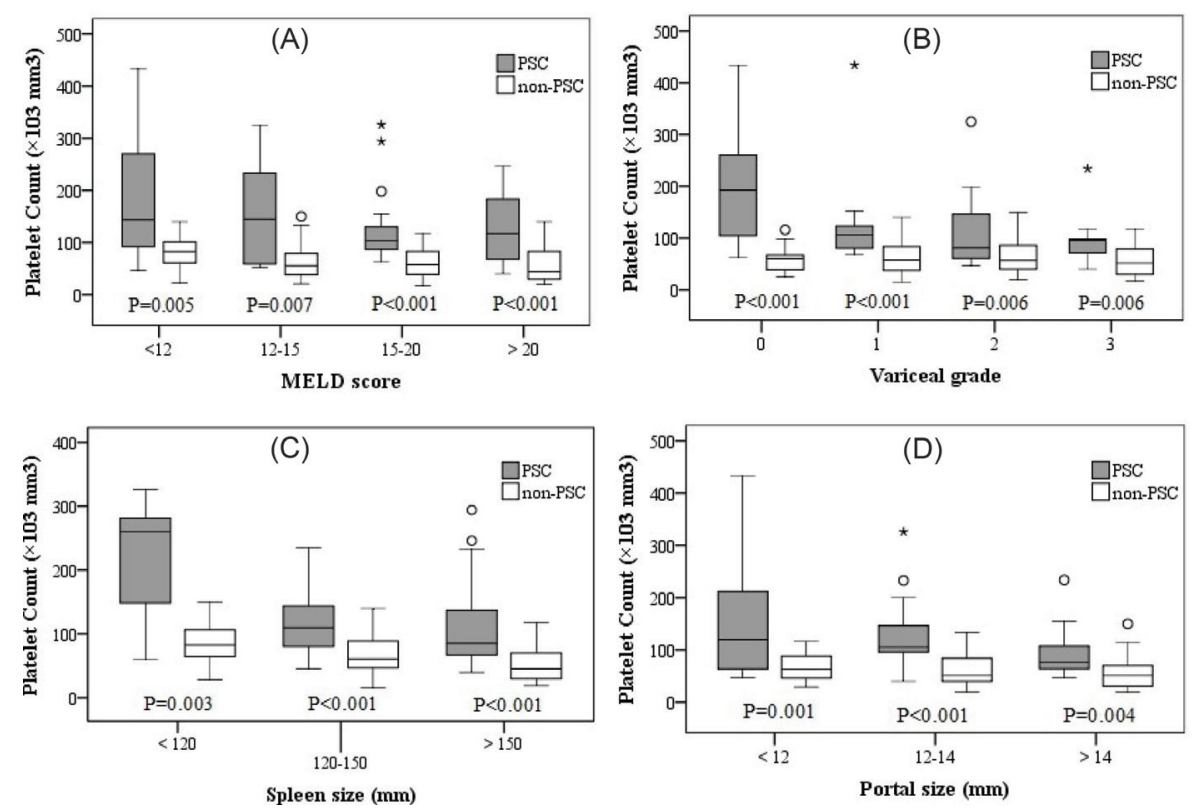

Figure 2. Comparison of Platelet Counts in Different (A) MELD Categories, (B) Variceal Grades, (C) Spleen Size Categories, and (D) Portal Vein Size Categories. 
compared to viral or alcoholic LC. ${ }^{33,34}$ In a study by Palareti et al, ${ }^{35}$ it was shown that patients with cholestatic liver disease are less prone to blood loss during liver transplantation. It has been hypothesized that the hypercoagulate condition and thrombosis at the levels of liver parenchyma microvasculature could play a role in the progression to fibrosis in the chronic liver disease. ${ }^{30}$ Our findings in the present study are in accordance with previous reports, which may provide new insight about the involvement of platelets in the pathogenesis of liver disease progression and fibrosis in PSC. Although we were unable to precisely explain the pathophysiologic background of increased levels of platelet count in PSC-C patients, several potential explanations for this finding could be mentioned.

Previous studies show that an increase in fibrinogen is well established as a causative factor for increasing ESR. ${ }^{36,37}$ Moreover, higher levels of fibrinogen have been previously described in cholestatic liver disease compared to other liver diseases. ${ }^{23,24,36}$ Therefore, elevated fibrinogen levels are associated with stable platelet counts in cirrhotic PSC patients and result in decreased platelet destruction. ${ }^{24}$ Another reason could be the increased levels of inflammatory markers such as ESR and CRP in PSC-C patients compared to patients with viral or alcoholic and non-alcoholic liver disease, which may lead to higher levels of platelets in PSC-C patients. ${ }^{24}$ The relationship between increased platelet counts and inflammatory disease have been also shown in other medical conditions. ${ }^{38-40}$ Although we did not measure fibrinogen levels in our survey, the levels of inflammatory markers such as ESR were significantly higher among PSC-C patients compared to non-PSC-C etiologies and this in turn may explain the reason for higher platelet counts in our PSC-C patients. The underlying reason for this discrepancy is unclear. PSC is a disease with recurrent cholangitis and it might be possible that inflammatory processes in the cholangitis events could play a major role in intervening different stages of thrombopoiesis and affecting platelet levels. In addition, it has been assumed that there is better preservation of parenchymal tissue in the less involved area of the liver in PSC. ${ }^{35}$ This fact could be due to the nonuniform nature of biliary stricture and beading in PSC, which subsequently results in higher levels of coagulation factors, as well as platelet counts in PSC-C patients.

Given the complex nature of PSC and lack of clear-cut diagnostic criteria, establishing a precise diagnosis of PSC is still problematic. Diagnosis of PSC is usually based on evidence of a cholestatic pattern of liver biochemistries in combination with typical imaging findings. ${ }^{41}$ Liver biopsy findings are not completely supportive of diagnosis, confirmation and staging of PSC. However, due to the invasive nature of liver biopsy, sampling error and high costs, the application of this procedure has been limited. ${ }^{42,43}$ Currently, the use of non-invasive diagnostic methodologies have been proposed, including serumbased markers or models in the assessment of prognosis of cirrhotic patients in various kinds of liver diseases. ${ }^{44-50}$ However, limited data exists regarding noninvasive diagnostic tests in terms of distinguishing between PSC-C and other cholestatic cirrhosis etiologies.

On the other hand, it should be noted that the findings presented in this study indicate the inability of predicting the severity of hepatic fibrosis among PSC patients based on the platelet-dependent models such as aspartate aminotransferase-to-platelet ratio index, gamma-glutamyl transferase-to-platelet ratio, fibrosis index based on the 4 factors, and red blood cell distribution width-to-platelet ratio. ${ }^{51-55}$ Therefore, in the PSC-C patients, this context of platelet count status should be considered in the application of models predicting progression to cirrhosis. Recently, in a large cohort of PSC patients from the United Kingdom (UK-PSC score), a new risk scoring model was developed in order to predict the outcome of patients diagnosed with PSC. ${ }^{56}$ They concluded that platelet counts along with other parameters such as bilirubin, albumin, hemoglobin, alkaline phosphatase, variceal bleeding and cholangiographic disease distribution have a better performance in risk assessment of PSC patients when compared to previous models. Future larger studies are needed to validate these models for assessment of progression of liver disease among PSC-C patients.

The present study had several limitations. First, the relatively small sample size of our study may have reduced the power to detect a significant correlation between platelet counts and PSC diagnosis. Second, we did not have an exact assessment of liver fibrosis and portal hypertension of study subjects by histology review and hepatic venous pressure gradient. Third, the wide range of the confidence interval of the relative risk of non-PSC-C diagnosis could be due to the possibility of sparse-data bias as previously described. ${ }^{57}$ Therefore, the results of the present study should be interpreted in the context of its limitations.

In summary, the findings of this cohort indicate that PSC-C patients presented with mild degrees of thrombocytopenia compared to other causes of liver disease. The reason for this discrepancy is unknown. Further studies are warranted to clarify the exact molecular mechanism and possible clinical implications of this finding in management of patients with PSC-C awaiting liver transplantation.

\section{Authors' Contribution}

MNT (Critical revision of the manuscript, Study concept and design), BM (Acquisition, analysis and interpretation of data, drafting of the manuscript), $\mathrm{AE}, \mathrm{FAA}, \mathrm{HJH}$ (participated in the data acquisition), MK (analysis, interpretation of data, statistical analysis), and AJ (Study concept and design, critical revision of the manuscript).

\section{Conflict of Interest Disclosures}

The authors have no conflict of interest to declare. 


\section{Ethical Statement}

The study protocol was approved by the ethics committee of Tehran University of Medical Science. Informed consent was obtained from all included patients. The study was conducted in accordance with the Declaration of Helsinki and other applicable guidelines, laws, and regulations.

\section{Funding}

This research has been supported by Tehran University of Medical Sciences \& health services (Grant No: 95-02-205-32311).

\section{Acknowledgements}

We would like to thank the liver transplantation research program nurses for their assistance in measurements and data management.

\section{Supplementary Materials}

Supplementary file 1 contains Figure S1.

\section{References}

1. Zhou WC, Zhang QB, Qiao L. Pathogenesis of liver cirrhosis. World J Gastroenterol. 2014;20(23):7312-24. doi:10.3748/ wjg.v20.i23.7312.

2. Qamar AA, Grace ND, Groszmann RJ, Garcia-Tsao G, Bosch $\mathrm{J}$, Burroughs AK, et al. Incidence, prevalence, and clinical significance of abnormal hematologic indices in compensated cirrhosis. Clin Gastroenterol Hepatol. 2009;7(6):689-95. doi:10.1016/j.cgh.2009.02.021.

3. Hayashi H, Beppu T, Shirabe K, Maehara Y, Baba H. Management of thrombocytopenia due to liver cirrhosis: a review. World J Gastroenterol. 2014;20(10):2595-605. doi:10.3748/wjg.v20.i10.2595.

4. McCormick PA, Murphy KM. Splenomegaly, hypersplenism and coagulation abnormalities in liver disease. Baillieres Best Pract Res Clin Gastroenterol. 2000;14(6):1009-31. doi:10.1053/bega.2000.0144

5. Violi F, Ferro D, Quintarelli C, Saliola M, Cordova C, Balsano F. Clotting abnormalities in chronic liver disease. Dig Dis. 1992;10(3):162-72. doi:10.1159/000171354.

6. Afdhal N, McHutchison J, Brown R, Jacobson I, Manns M, Poordad F, et al. Thrombocytopenia associated with chronic liver disease. J Hepatol. 2008;48(6):1000-7. doi:10.1016/j. jhep.2008.03.009.

7. Giannini EG. Review article: thrombocytopenia in chronic liver disease and pharmacologic treatment options. Aliment Pharmacol Ther. 2006;23(8):1055-65. doi:10.1111/j.13652036.2006.02889.x.

8. Bashour FN, Teran JC, Mullen KD. Prevalence of peripheral blood cytopenias (hypersplenism) in patients with nonalcoholic chronic liver disease. Am J Gastroenterol. 2000;95(10):29369. doi:10.1111/j.1572-0241.2000.02325.x.

9. Weksler BB. Review article: the pathophysiology of thrombocytopenia in hepatitis $C$ virus infection and chronic liver disease. Aliment Pharmacol Ther. 2007;26 Suppl 1:13-9. doi:10.1111/j.1365-2036.2007.03512.x.

10. Rios R, Sangro B, Herrero I, Quiroga J, Prieto J. The role of thrombopoietin in the thrombocytopenia of patients with liver cirrhosis. Am J Gastroenterol. 2005;100(6):1311-6. doi:10.1111/j.1572-0241.2005.41543.x.

11. Lee CM, Leung TK, Wang HJ, Lee WH, Shen LK, Liu JD, et al. Evaluation of the effect of partial splenic embolization on platelet values for liver cirrhosis patients with thrombocytopenia. World J Gastroenterol. 2007;13(4):619-22.

12. Noguchi H, Hirai K, Aoki Y, Sakata K, Tanikawa K. Changes in platelet kinetics after a partial splenic arterial embolization in cirrhotic patients with hypersplenism. Hepatology. 1995;22(6):1682-8.

13. Peck-Radosavljevic M, Wichlas M, Zacherl J, Stiegler G, Stohlawetz P, Fuchsjager M, et al. Thrombopoietin induces rapid resolution of thrombocytopenia after orthotopic liver transplantation through increased platelet production. Blood. 2000;95(3):795-801.

14. Kajihara $M$, Okazaki $Y$, Kato S, Ishii H, Kawakami Y, Ikeda $Y$, et al. Evaluation of platelet kinetics in patients with liver cirrhosis: similarity to idiopathic thrombocytopenic purpura. J Gastroenterol Hepatol. 2007;22(1):112-8. doi:10.1111/ j.1440-1746.2006.04359.x.

15. Pereira J, Accatino L, Alfaro J, Brahm J, Hidalgo P, Mezzano D. Platelet autoantibodies in patients with chronic liver disease. Am J Hematol. 1995;50(3):173-8.

16. Aref S, Sleem T, El Menshawy N, Ebrahiem L, Abdella D, Fouda $M$, et al. Antiplatelet antibodies contribute to thrombocytopenia associated with chronic hepatitis C virus infection. Hematology. 2009;14(5):277-81. doi:10.1179/102453309x439818.

17. Wang CS, Yao WJ, Wang ST, Chang TT, Chou P. Strong association of hepatitis $\mathrm{C}$ virus (HCV) infection and thrombocytopenia: implications from a survey of a community with hyperendemic HCV infection. Clin Infect Dis. 2004; 39(6):790-6. doi:10.1086/423384.

18. Poynard T, Bedossa P. Age and platelet count: a simple index for predicting the presence of histological lesions in patients with antibodies to hepatitis C virus. METAVIR and CLINIVIR Cooperative Study Groups. J Viral Hepat. 1997;4(3):199-208.

19. Liu F, Zhou H, Cao L, Guo Z, Dong C, Yu L, et al. Risk of reduced platelet counts in patients with nonalcoholic fatty liver disease (NAFLD): a prospective cohort study. Lipids Health Dis. 2018;17(1):221. doi:10.1186/s12944-018-08657 .

20. Chapman R, Fevery J, Kalloo A, Nagorney DM, Boberg KM, Shneider B, et al. Diagnosis and management of primary sclerosing cholangitis. Hepatology. 2010;51(2):660-78. doi:10.1002/hep.23294.

21. Isayama H, Tazuma S, Kokudo N, Tanaka A, Tsuyuguchi T, Nakazawa T, et al. Clinical guidelines for primary sclerosing cholangitis 2017. J Gastroenterol. 2018;53(9):1006-34. doi:10.1007/s00535-018-1484-9.

22. Karlsen TH, Folseraas T, Thorburn D, Vesterhus M. Primary sclerosing cholangitis - a comprehensive review. J Hepatol. 2017;67(6):1298-323. doi:10.1016/j.jhep.2017.07.022.

23. Ben-Ari Z, Panagou M, Patch D, Bates S, Osman E, Pasi J, et al. Hypercoagulability in patients with primary biliary cirrhosis and primary sclerosing cholangitis evaluated by thrombelastography. J Hepatol. 1997;26(3):554-9.

24. Pihusch R, Rank A, Gohring P, Pihusch M, Hiller E, Beuers U. Platelet function rather than plasmatic coagulation explains hypercoagulable state in cholestatic liver disease. J Hepatol. 2002;37(5):548-55

25. Lindor KD, Kowdley KV, Harrison ME. ACG Clinical Guideline: Primary Sclerosing Cholangitis. Am J Gastroenterol. 2015; 110(5):646-59. doi:10.1038/ajg.2015.112.

26. Lee YM, Kaplan MM. Primary sclerosing cholangitis. $N$ Engl J Med. 1995;332(14):924-33. doi:10.1056/ nejm 199504063321406 .

27. EASL Clinical Practice Guidelines: management of cholestatic liver diseases. J Hepatol. 2009;51(2):237-67. doi:10.1016/j. jhep.2009.04.009.

28. World Medical Association Declaration of Helsinki: ethical principles for medical research involving human subjects. Jama. 2013;310(20):2191-4. doi:10.1001/jama.2013.281053.

29. Beppu K, Inokuchi K, Koyanagi N, Nakayama S, Sakata $\mathrm{H}$, Kitano S, et al. Prediction of variceal hemorrhage by esophageal endoscopy. Gastrointest Endosc. 1981;27(4):2138.

30. Anstee QM, Dhar A, Thursz MR. The role of hypercoagulability in liver fibrogenesis. Clin Res Hepatol Gastroenterol. 2011; 35(8-9):526-33. doi:10.1016/j.clinre.2011.03.011.

31. Tejima K, Masuzaki R, Ikeda H, Yoshida H, Tateishi R, Sugioka 
$\mathrm{Y}$, et al. Thrombocytopenia is more severe in patients with advanced chronic hepatitis $C$ than $B$ with the same grade of liver stiffness and splenomegaly. J Gastroenterol. 2010; 45(8):876-84. doi:10.1007/s00535-010-0233-5.

32. Ikarashi Y, Kodama K, Taniai M, Hashimoto E, Tokushige K. The clinical difference in the platelet counts between liver cirrhosis with nonalcoholic fatty liver disease and hepatitis C virus. Intern Med. 2018;57(8):1065-70. doi:10.2169/ internalmedicine.9853-17

33. Gores GJ, Wiesner RH, Dickson ER, Zinsmeister AR, Jorgensen RA, Langworthy A. Prospective evaluation of esophageal varices in primary biliary cirrhosis: development, natural history, and influence on survival. Gastroenterology. 1989;96(6):1552-9.

34. Segal H, Cottam S, Potter D, Hunt BJ. Coagulation and fibrinolysis in primary biliary cirrhosis compared with other liver disease and during orthotopic liver transplantation. Hepatology. 1997;25(3):683-8. doi:10.1002/hep.510250332.

35. Palareti G, Legnani C, Maccaferri M, Gozzetti G, Mazziotti A, Martinelli G, et al. Coagulation and fibrinolysis in orthotopic liver transplantation: role of the recipient's disease and use of antithrombin III concentrates. S. Orsola Working Group on Liver Transplantation. Haemostasis. 1991;21(2):68-76. doi:10.1159/000216206.

36. Holley L, Woodland N, Hung WT, Cordatos K, Reuben A. Influence of fibrinogen and haematocrit on erythrocyte sedimentation kinetics. Biorheology. 1999;36(4):287-97.

37. Bain BJ. Some influences on the ESR and the fibrinogen level in healthy subjects. Clin Lab Haematol. 1983;5(1):45-54.

38. Morrell CN, Sun H, Swaim AM, Baldwin WM, 3rd. Platelets an inflammatory force in transplantation. Am J Transplant. 2007;7(11):2447-54. doi:10.1111/j.16006143.2007.01958.x.

39. Danese S, de la Motte C, Sturm A, Vogel JD, West GA, Strong $\mathrm{SA}$, et al. Platelets trigger a CD40-dependent inflammatory response in the microvasculature of inflammatory bowel disease patients. Gastroenterology. 2003;124(5):1249-64.

40. Smith TL, Weyrich AS. Platelets as central mediators of systemic inflammatory responses. Thromb Res. 2011;127(5):391-4. doi:10.1016/j.thromres.2010.10.013.

41. Yimam KK, Bowlus CL. Diagnosis and classification of primary sclerosing cholangitis. Autoimmun Rev. 2014;13(4-5):445-50. doi:10.1016/j.autrev.2014.01.040

42. Burak KW, Angulo P, Lindor KD. Is there a role for liver biopsy in primary sclerosing cholangitis? Am J Gastroenterol. 2003;98(5):1155-8. doi:10.1111/j.1572-0241.2003.07401.x.

43. Olsson R, Hagerstrand I, Broome U, Danielsson A, Jarnerot G, Loof L, et al. Sampling variability of percutaneous liver biopsy in primary sclerosing cholangitis. J Clin Pathol. 1995;48(10):933-5

44. de Vries EM, Wang J, Williamson KD, Leeflang MM, Boonstra K, Weersma RK, et al. A novel prognostic model for transplant-free survival in primary sclerosing cholangitis. Gut. 2018;67(10):1864-9. doi:10.1136/gutjnl-2016-313681.
45. Haj M, Hart M, Rockey DC. Development of a novel clinical staging model for cirrhosis using the Nationwide Inpatient Sample. J Investig Med. 2018;66(6):992-7. doi:10.1136/jim2018-000709.

46. Lin W, Zhang J, Liu X, Liu H, He J, Li M, et al. A Dynamic Model for Predicting Outcome in Patients with HBV Related AcuteOn-Chronic Liver Failure. Ann Hepatol. 2018;17(3):392-402. doi:10.5604/01.3001.0011.7383.

47. Benedeto-Stojanov D, Nagorni A, Bjelakovic G, Stojanov D, Mladenovic B, Djenic N. The model for the end-stage liver disease and Child-Pugh score in predicting prognosis in patients with liver cirrhosis and esophageal variceal bleeding. Vojnosanit Pregl. 2009;66(9):724-8.

48. Castera L. Noninvasive assessment of liver fibrosis. Dig Dis. 2015;33(4):498-503. doi:10.1159/000374097.

49. Li B, Zhang L, Zhang Z, Yan G, Zhu L, Lu W, et al. A noninvasive indicator for the diagnosis of early hepatitis B virus-related liver fibrosis. Eur J Gastroenterol Hepatol. 2019;31(2):218223. doi:10.1097/meg.0000000000001281.

50. Lu XJ, Li XH, Yuan ZX, Sun HY, Wang XC, Qi X, et al. Assessment of liver fibrosis with the gamma-glutamyl transpeptidase to platelet ratio: a multicentre validation in patients with HBV infection. Gut. 2018;67(10):1903-4. doi:10.1136/gutjnl-2017-315299.

51. Chen B, Ye B, Zhang J, Ying L, Chen Y. RDW to Platelet Ratio: A Novel Noninvasive Index for Predicting Hepatic Fibrosis and Cirrhosis in Chronic Hepatitis B. PLoS One. 2013; 8(7):e68780. doi:10.1371/journal.pone.0068780.

52. Liu CH, Liang CC, Liu CJ, Hsu SJ, Lin JW, Chen SI, et al. The ratio of aminotransferase to platelets is a useful index for predicting hepatic fibrosis in hemodialysis patients with chronic hepatitis C. Kidney Int. 2010;78(1):103-9. doi:10.1038/ki.2010.74.

53. Vallet-Pichard A, Mallet V, Nalpas B, Verkarre V, Nalpas A, Dhalluin-Venier $V$, et al. FIB-4: an inexpensive and accurate marker of fibrosis in $\mathrm{HCV}$ infection. comparison with liver biopsy and fibrotest. Hepatology. 2007;46(1):32-6. doi:10.1002/hep.21669.

54. Jain P, Tripathi BK, Gupta B, Bhandari B, Jalan D. Evaluation of Aspartate Aminotransferase-to-Platelet Ratio Index as a NonInvasive Marker for Liver Cirrhosis. J Clin Diagn Res. 2015; 9(11):Oc22-4. doi:10.7860/jcdr/2015/13944.6814.

55. Lee J, Kim MY, Kang SH, Kim J, Uh Y, Yoon KJ, et al. The gamma-glutamyl transferase to platelet ratio and the FIB-4 score are noninvasive markers to determine the severity of liver fibrosis in chronic hepatitis B infection. Br J Biomed Sci. 2018;75(3):128-32. doi:10.1080/09674845.2018.1459147.

56. Goode EC, Clark AB, Mells GF, Srivastava B, Spiess K, Gelson WTH, et al. Factors associated with outcomes of patients with primary sclerosing cholangitis and development and validation of a risk scoring system. 2019;69(5):2120-35. doi:10.1002/hep.30479.

57. Greenland S, Mansournia MA, Altman DG. Sparse data bias: a problem hiding in plain sight. Bmj. 2016;352:i1981. doi:10.1136/bmj.i1981. 